\title{
Modeling of Blumlein circuit and calculation of resistance and inductance of Laser Plasma
}

\author{
Caifeng Zhao, Jianqiang Wu \\ Electrical Engineering Department, Harbin Institute of Technology, Harbin, China
}

Email address:

seethescene@163.com (Caifeng Zhao),wjq@hit.edu.cn (Jianqiang Wu)

To cite this article:

Caifeng Zhao, Jianqiang Wu. Modeling of Blumlein Circuit and Calculation of Resistance and Inductance of Laser Plasma. International Journal of Science, Technology and Society. Vol. 2, No. 6, 2014, pp. 196-200. doi: 10.11648/j.ijsts.20140206.16

\begin{abstract}
Laser plasma discharge circuit can be simulated by Pspice through practical measuring result. We find that the circuit model of laser plasma should be simulated by a inductance in series with a resistor, furthermore in parallel with a capacitor. The value of the capacitor affect the oscillation frequency of the laser output voltage. The smaller the value of the equivalent capacitor, the less the rise time of the pulse voltage of the laser chamber. Also the rise time of the pulsed voltage of laser can be less when the stray inductance and transmission line capacitance are reduced. It is also found the time -invariant inductance is dependent on the total charge in the discharge area, the time-invariant inductance is dependent on the electric field in the laser chamber.
\end{abstract}

Keywords: Blumlein, Pspice, Spark Gap, Laser Plasma, Curve Proximity, Matlab

\section{Introduction}

It is necessary to generate high breakdown voltage for pumping lasers by discharge. When a pulsed voltage is applied to a kind of gas medium in a laser chamber, the gas don't experience voltage breakdown instantaneously but after a short time frequent discharge. The delay time depends on the exerted voltage, the electrode structure, electrode spacing, charged gas gradients, pressure, effect of prebreakdown ionization etc. When the voltage generating time is shorter than the breakdown time, the laser can operate on a static higher breakdown voltage.

Because the life time of a sulphur molecule is very short, a rapid pulsed discharge pump is necessary. In order to obtain a pulsed current of high amplitude with steep rise edge, we need to choose a suitable pulsed circuit. Comparing generally used transmission circuit, double pulsed thyristor trigger circuit and Blumlein circuit, we found Blumlein circuit an ideal choice due to its double voltage output and its short current rising time.

\section{Theory Analysis}

A Blumlein circuit for $S_{2}$ exciting is shown in Fig. 1. In this figure, $\mathrm{C}_{\mathrm{S}}$ is a storage capacitor, $\mathrm{C}_{\mathrm{P}}$ is a transmission capacitor which is in parallel with a spark gap switch $\mathrm{K}_{1}$, $\mathrm{K}_{2}$ is a laser discharge chamber which is in parallel with a bigger inductor $\mathrm{L}$.

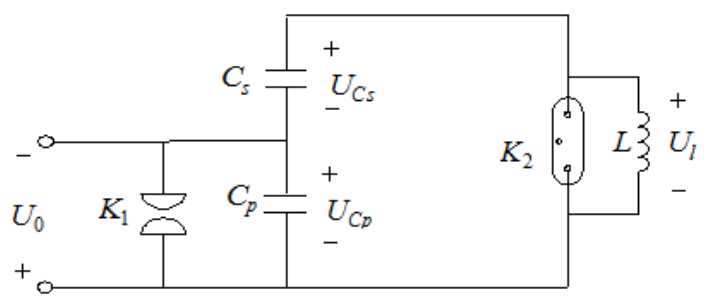

Fig. 1. Blumlein Circuit

In the experiment, a directing voltage is applied across the the voltage direction shown in Fig. 1, the voltage across $\mathrm{C}_{\mathrm{S}}$, $\mathrm{C}_{\mathrm{P}}$ is $-U_{\mathrm{o}}, U_{\mathrm{o}}$ respectively. When the directing voltage is moved off, a negative voltage is applied to the trigger electrode of the spark gap switch and cause it breakdown. Generally when a spark switch is brokendown, it combines $\mathrm{C}_{\mathrm{P}}$ to form a RLC oscillator since it is regarded as an equivalent inductor in series with a resistor, and the inductor $\mathrm{L}$ acts like an open circuit because of the high oscillating frequency. When the voltage direction of $\mathrm{C}_{\mathrm{P}}$ change inversely, worked with the voltage $U_{0}$ of $\mathrm{C}_{\mathrm{S}}$, the laser chamber will be breakdown, then the laser plasma can be 
equivalent with a inductor in series with a resistor.

The equivalent RLC oscillating circuit before the laser chamber is brokendown is shown in Fig. 2. In the figure, the inductance of the spark gap switch and the stray inductance in the circuit is expressed as $L_{S}$, the resistance of the spark gap switch is represented as $r_{S}$. We can calculate the oscillating frequency through the voltage equation to determine the changing speed of the voltage.

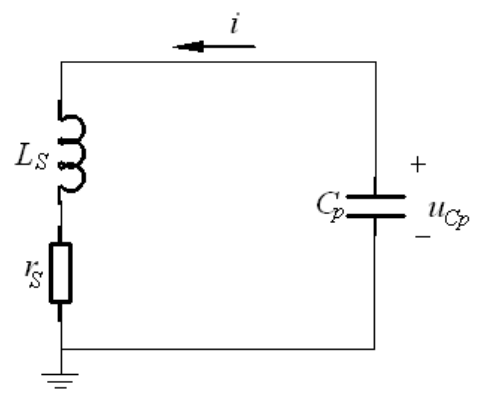

Fig. 2. Spark gap

the voltage equation in Fig. 2 is

$$
L_{S} \frac{d i}{d t}+r_{S} i+\frac{1}{C_{P}} \int_{0}^{t} i d t=u_{C_{P}}(0)
$$

in the expression above, $\omega$ is the oscillating angular frequency

$$
\omega=\sqrt{\frac{1}{L_{S} C_{P}}-\left(\frac{r_{S}}{2 L_{S}}\right)^{2}}
$$

$b$ is damping coefficient

$$
b=\frac{r_{S}}{2 L_{S}}
$$

the voltage across the laser chamber is

$$
u_{L}=U_{0}\left(1-\frac{1}{\omega \sqrt{L_{S} C_{p}}} e^{-b t} \cos (\omega t+\varphi)\right)
$$

If the equivalent laser plasma inductance and resistance are expressed as $L_{L}$ and $r_{L}$, then the equivalent circuit after the laser chamber is brokendown is shown in Fig. 3.

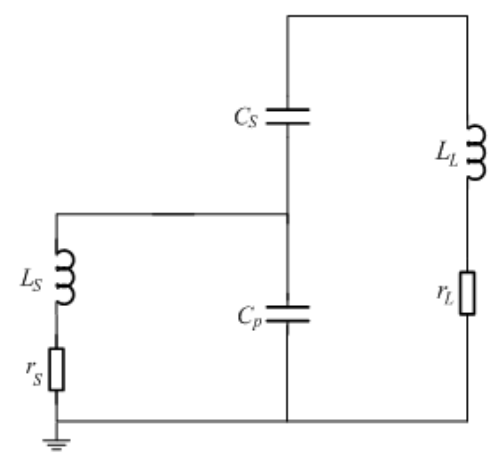

Fig. 3. The equivalent laser chamber circuit

\section{Experimental Results of Laser Discharge Circuit}

In the experiment, the transmission capacitor and the storage capacitor are composed of groups of seven and four Japanese capacitor with less inductance respectively, so $C_{P}=3.5 n F, C_{S}=2 n F$. The discharge voltage is measured by high voltage probe Tektronix 6015 connected with digital oscilloscope TEK380. In the experiment, 2 atmosphere air is pumped into the spark gap switch, 16.0Kpa neon is pumped into the laser chamber, the voltage waveforms are shown in Fig. 4 and Fig. 5. respectively. (The waveform below is measured by capacitor voltage divider)

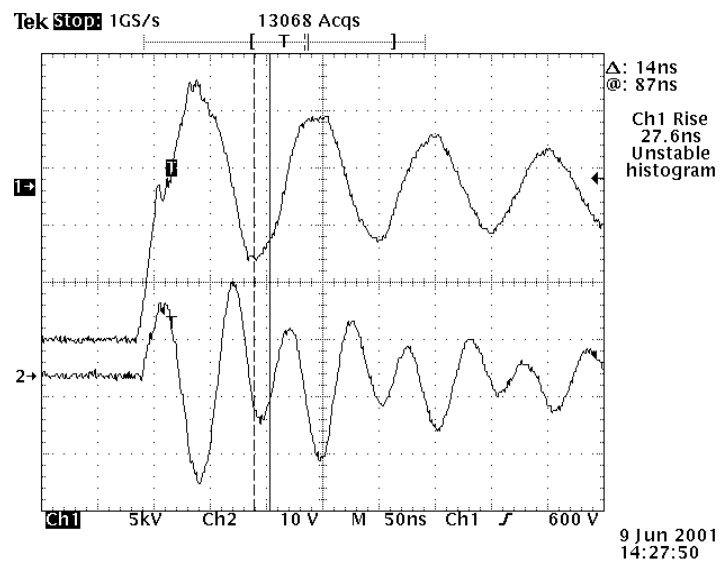

Fig. 4. The voltage waveform of the spark gap after breakdown

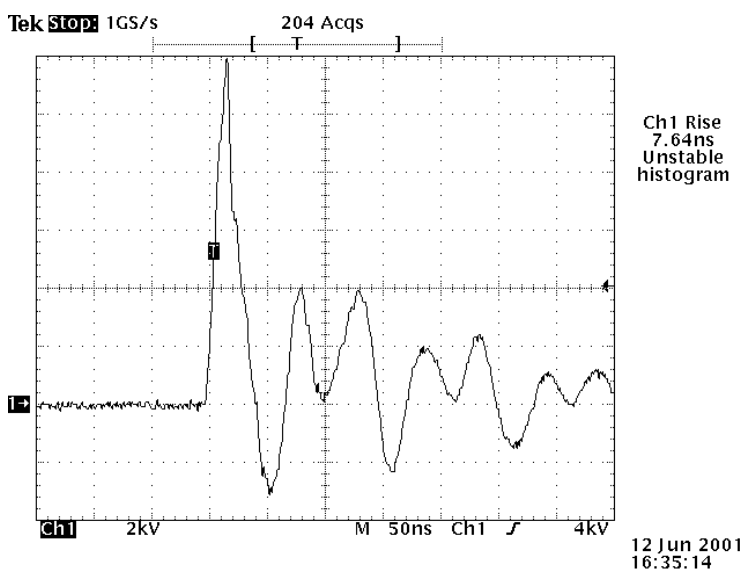

Fig. 5. The voltage waveform of the laser after breakdown

We can see that the negative charge voltage is $13 \mathrm{KV}$, the reverse voltage is $10 \mathrm{KV}$, the pulsed voltage rise time is $27.6 \mathrm{~ns}$. In the main circuit, the laser chamber is brokendown at the voltage of $12 \mathrm{KV}$, and the pulsed voltage rise time is $7.64 \mathrm{~ns}$.

For no wire in the main circuit, the pulse current can't be measured through a titanium film in series with the circuit. The size of the Titanium film is $30 \mathrm{~cm}$ in length., $1 \mathrm{~cm}$ in width, $30 \mu \mathrm{m}$ in thick, For its resistivity is

$$
R=\rho \frac{l}{S}=42.0 \times 10^{-2} \times \frac{0.3}{10 \times 30 \times 10^{-3}}=0.42 \Omega
$$




\section{Modeling of Blumlein Circuit and Its Calculation of the Resistance and Inductance of Laser Plasma}

The circuit we draw in PSPICE is shown in Fig. 6. We find that when the laser circuit part (on the right) and the spark gap circuit part (on the left) have the same parameters in quantity, the oscillating frequency are the same, and the rule of the waveform is complicated. Obviously the oscillating waveform of the spark gap is underdamped, so the inductance in the laser circuit is very high, and acts like an open circuit to the spark gap circuit. When the stray inductance is several hundreds of nanohenry, their effect on the spark gap can be neglected.

The equivalent inductance of the spark gap can be determined by the voltage oscillating angular frequency in Eq. (4)

$$
\omega \approx \sqrt{\frac{1}{L_{S} C_{P}}}
$$

From Fig. 4 we know that the actual voltage oscillating cycle is about $105 \mathrm{~ns}$, the equivalent spark gap inductance L2 is $87 \mathrm{nH}$. The equivalent resistance of the spark gap is determined by the damping coefficient of Eq. (3), consulting the first wave apex $10 \mathrm{kV}$, the simulated equivalent resistance $\mathrm{R} 11$ is $0.61 \Omega$.

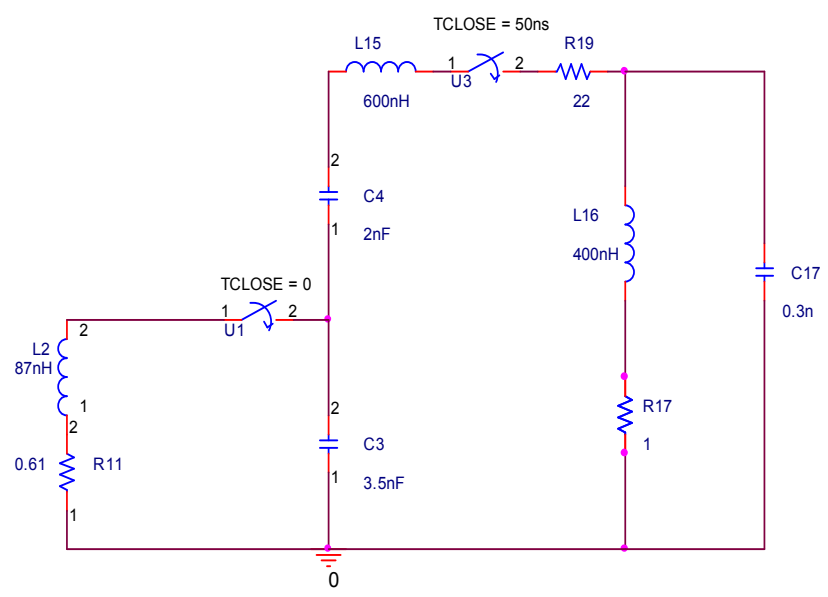

Fig. 6. The equivalent laser circuit in Pspice after breakdown

The simulated waveform of spark gap voltage versus time is shown in Fig. 7.

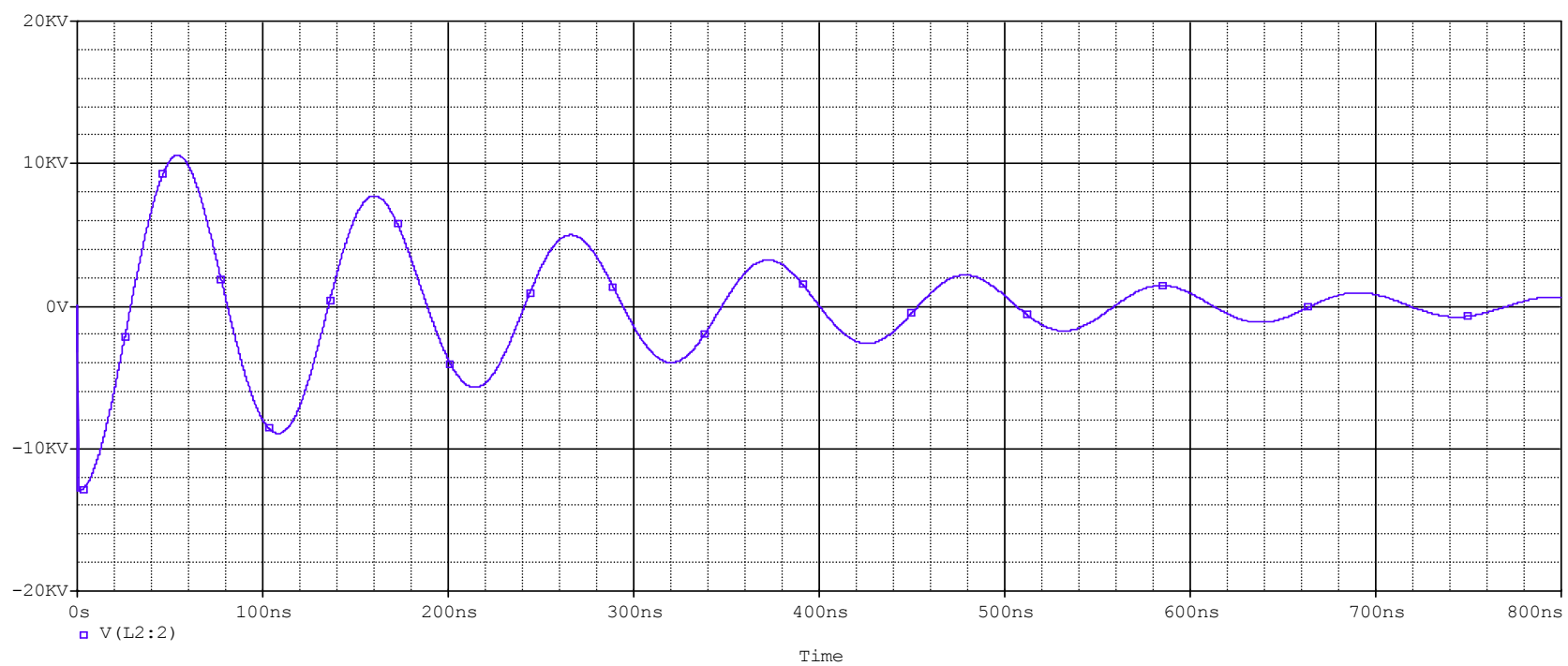

Fig. 7. The simulated waveform of spark gap voltage versus time

When the laser plasma equivalent resistance R17 is set to be $1 \Omega^{[3]}$, the equivalent inductance is $1000 \mathrm{nH}$, the wave apex of the simulated spark gap voltage decrease gradually. From Fig. 5 , we know that the breakdown voltage of the laser chamber is not equal to the sum of the inverse voltage of the spark gap and the initial voltage of the storage capacitor, and there exist stray inductance except the inductance of laser plasma.

Changing the inductance ratio of L15 and L16, we can make the breakdown voltage across the laser plasma to be $12 \mathrm{KV}$.

The value of stray inductance and the laser plasma inductance is $600 \mathrm{nH}$ and $400 \mathrm{nH}$ respectively. If only the stray inductance and the laser plasma inductance are considered, the voltage oscillating cycle keep the same with that of the spark gap but not the same as the parallel apex structure in Fig. 5, so we need to correct the modeling of the laser chamber. When a capacitor is in parallel with the laser chamber, if the capacitance is small, multimodal structure will occur and the voltage output waveform of the spark gap won't change. The modeling equivalent capacitance $\mathrm{C} 17$ is $0.3 \mathrm{nH}$.

By changing the value of R19, the parallel apex structure approaches to the actual waveform. The modeling stray resistance in the circuit is $22 \Omega$.

A delay switch can be used to control the laser chamber on or off. The switch parameter time TCLOSE depends on the inverse time of the spark gap voltage, and is set to be 50ns in Fig.5. The value of TTRAN is set to be $0.00001 \mathrm{~ns}$, the value of RCLOSED is set to be $0.00001 \Omega$. The modeling voltage 
waveform of the laser plasma after conducting is shown in Fig. $\quad 8$.

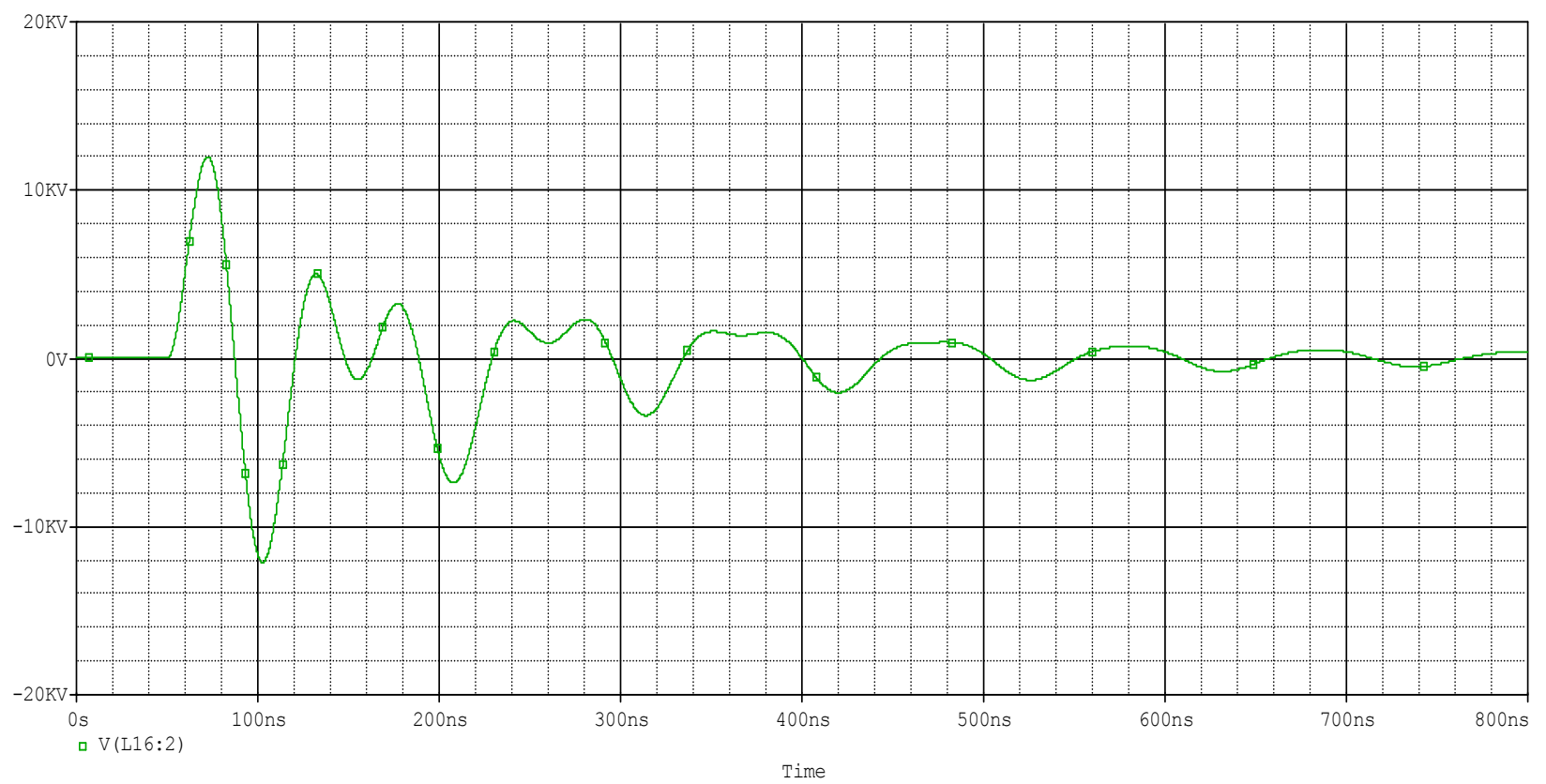

Fig. 8. Modeling voltage waveform of the laser chamber after conducting

Also the variation of the resistance and inductance of laser plasma at initial stage can be measured through the breakdown voltage and current waveforms of the laser chamber. The plasma resistance at initial stage can be given as ${ }^{[4]}$

$$
R=\frac{\sqrt{P} d}{\sqrt{2 a \int_{0}^{t} i^{2} d t}}
$$

In the equation, $\mathrm{P}$ is the number of atmosphere, $\mathrm{d}$ is the electrode distance which is in centimeter. The value of $a$ depends on the kind of gas and is in atmosphere $\mathrm{cm}^{2} / \mathrm{s}$. volt ${ }^{2}$

In the experiment, $16.0 \mathrm{Kpa}$ neon is pumped into a laser chamber, then $a$ is 30 in the equation (5), and the measured electrode spacing is $0.78 \mathrm{~cm}$, the breakdown current waveforms of the laser chamber are shown in Fig.9.

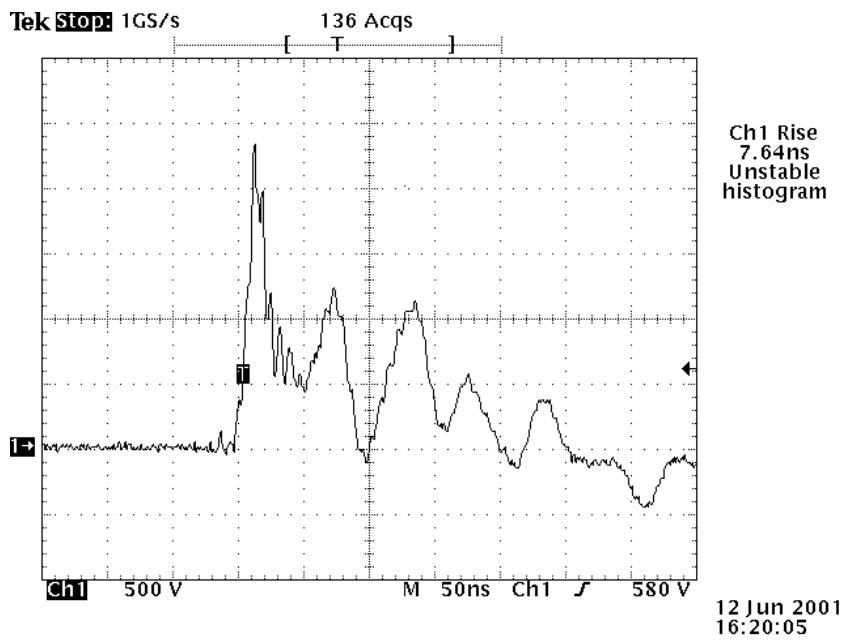

Fig. 9. The current waveform in the circuit
From Fig.9, the current function at initial phase can be simply expressed as

$i_{1}=2738.1+2738.1 \sin \left(0.2094 \times 10^{9} t-\frac{\pi}{2}\right) \quad$ when $0 \leq \mathrm{t} \leq 15 \mathrm{~ns}$

Substituting eq.(6) into eq.(5), we use Matlab to perform curve proximity every $1 \mathrm{~ns}$, then get the variation of laser plasma resistance versus current and time respectively. Fig.10 and Fig.11 shows that the resistance decrease rapidly with time and approaches $0.1 \Omega$.

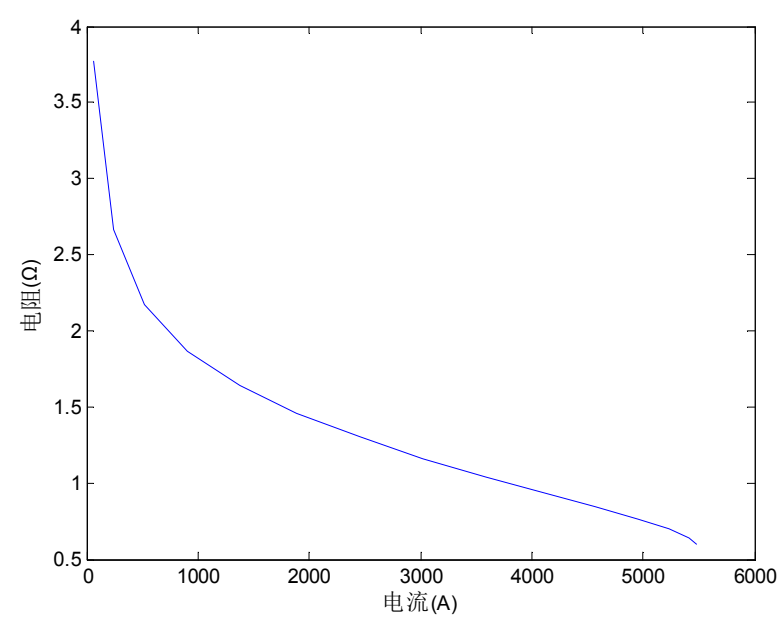

Fig. 10. The laser plasma resistance versus current at initial phase 


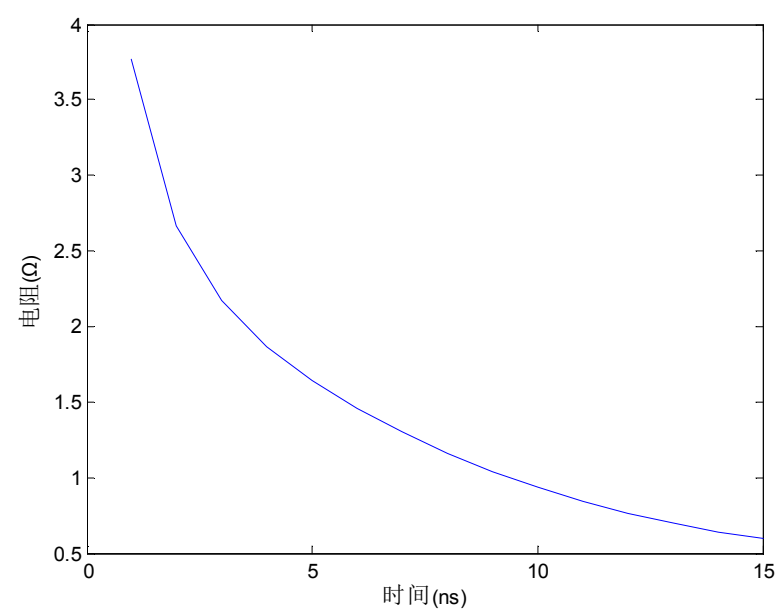

Fig. 11. The laser plasma resistance versus time at initial phase

From Fig.5 the breakdown voltage at initial time can be similarly expressed as

$$
u=4500+7500 \sin \left(0.0898 \times 10^{9} t+\frac{\pi}{2}\right)
$$

The voltage equation can be written as

$$
u=\frac{d(L i)}{d t}+R i=L \frac{d i}{d t}+i \frac{d L}{d t}+R i
$$

In the equation, $\mathrm{L}$ is the equivalent inductance of laser plasma. From the above relationship, we proceed curve proximity every $1 \mathrm{~ns}$, then we get

$$
L_{n}=\frac{\left(u_{n}-R_{n} i_{n}\right) \Delta t+i_{n} L_{n-1}}{2 i_{n}-i_{n-1}}
$$

We use MATLAB to calculate the value of $L$, then get the variation of laser plasma inductance versus current and time respectively. Fig.12 and Fig.13 shows that the inductance decrease rapidly with time and approaches $29 \mathrm{nH}$.

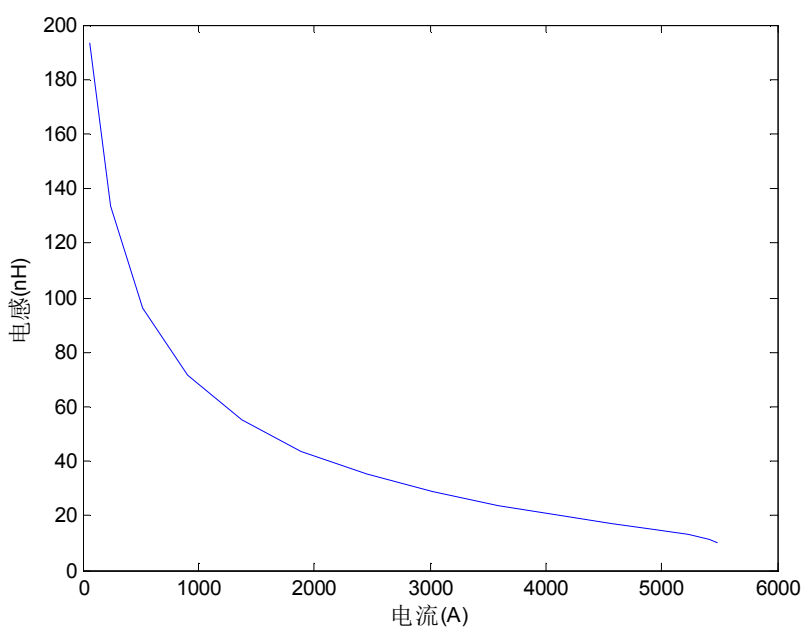

Fig. 12. The laser plasma inductance versus current at initial phase

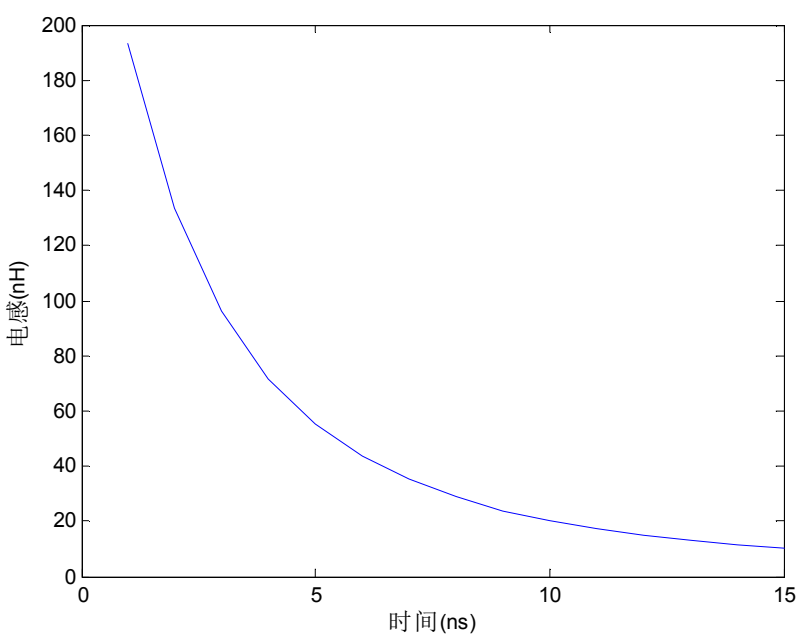

Fig. 13. The laser plasma inductance versus time at initial phase

\section{Acknowledgements}

By modeling Blumlein circuit through software Pspice and Matlab, we find that the circuit model of a laser chamber depicted to be a inductance in series with a resistance is not enough, it is reasonable to set up a parallel connection with a capacitor. From Eq. (2). We know that the oscillating cycle of the spark gap voltage, and furthermore the rising time of the laser chamber voltage will be shorten if we decrease the transmission capacitance or the stray inductance in the circuit. In addition, when the equivalent capacitance of the laser chamber is small, or the stray inductance in the circuit become smaller, the rising edge of the pulsed voltage of the laser chamber will become steep. Furthermore, a more easy way of calculating nonlinear resistance and inductance at discharge initial phase, and a reasonable explanation has been given.

\section{References}

[1] D. A. Peterson and D. R. Crosley, Hanle Effect of Lifetime Measurements on Selectively Excited Diatomic Sulfur. J. Chem. Phys. 1973, 159 (4): 1933-1941

[2] Wang Fuchun, Or CAD 9.0 Concise Course. Chinese mechanical publishing house. 2000, 9, First Edition.

[3] William D. Greason, Methodology to study the resistance of spark discharges. IEEE. Transactions on Industry Application, vol.35, no.2, pp.359, March/April 1999

[4] Rompe, R., Weizel W.: Ueber das Toeplersche Funkengesetz.Z.phys. 122, 636-639 (1944) 